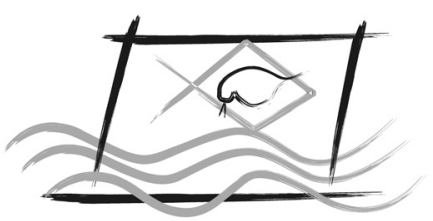

J. Braz. Soc. Ecotoxicol., v. 7, n. 1, 2012,

doi: $10.5132 / j b s e .2012 .01 .005$

ECOTOX - BRASIL

\title{
Toxicidade aguda e risco ambiental de surfactantes agrícolas para o guaru Phalloceros caudimaculatus (Pices: Poecilidae)
}

\author{
S.P. Carraschi ${ }^{1,2}$, L.A.V. LunA ${ }^{2}$, A.N. Neto ${ }^{2}$, A.C.F. Gírio², C. CruZ² \& R.A. Pitelli ${ }^{2}$ \\ ${ }^{1}$ Centro de Aquicultura da Unesp/Caunesp, Câmpus de Jaboticabal, Brasil \\ ${ }^{2}$ Núcleo de Estudos e Pesquisas Ambientais em Matologia, Faculdade de Ciências Agrárias e Veterinárias da UNESP, Câmpus de \\ Jaboticabal, São Paulo, Brasil.
}

(Received August 26, 2009; Accept November 04, 2011)

\begin{abstract}
Resumo
Os surfactantes são moléculas anfipáticas que promovem a redução da tensão superficial da água. Os objetivos foram estimar CL(I) $)_{50}$;96h; determinar o risco ambiental; avaliar a qualidade da água e os sinais de intoxicação durante os testes com os surfactantes Agral $^{\circledR}$, Aterbane ${ }^{\circledR}$ BR, Ag-Bem ${ }^{\circledR}$, Energic $^{\circledR}$, Fixade $^{\circledR}$ e Gotafix ${ }^{\circledR}$ para o guaru (Phalloceros caudimaculatus). Os animais foram expostos aos surfactantes e a um tratamento controle, em delineamento inteiramente casualizado, com três repetições. Para caracterização do risco ambiental foram utilizados os dados da concentração ambiental estimada (CAE) e da $\mathrm{CL}(\mathrm{I})_{50} ; 96 \mathrm{~h}$ de cada surfactante, classificando-os de acordo com o coeficiente de risco. A CL(I) $)_{50} ; 96 \mathrm{~h}$ do Agral $^{\circledR}$ foi $4,33 \mathrm{mgL}^{-1}$; do Aterbane ${ }^{\circledR} \mathrm{BR} 5,81 \mathrm{mgL}^{-1}$; do Energic ${ }^{\circledR} 3,93 \mathrm{mgL}^{-1}$; do Gotafix ${ }^{\circledR} 3,40 \mathrm{mgL}^{-1}$; do Fixade ${ }^{\circledR} 4,06 \mathrm{mgL}^{-1}$ e do Ag-Bem ${ }^{\circledR} 22,19 \mathrm{mgL}^{-}$ 1. A qualidade da água não foi alterada pela presença dos surfactantes. Os sinais de intoxicação apresentados foram: aumento do batimento opercular; perda da capacidade de arfagem; natação errática e espasmos. O Ag-Bem ${ }^{\circledR}$ pode ser considerado como pouco tóxico e os demais, moderadamente tóxicos. O Aterbane ${ }^{\circledR} \mathrm{BR}$, o $\mathrm{Agral}^{\circledR}$, o Ag-Bem ${ }^{\circledR}$ e o $\mathrm{Gotafix}^{\circledR}$ são considerados de risco com possibilidade de efeito adverso e o Energic $^{\circledR}$ e o Fixade $^{\circledR}$ de risco com probabilidade de efeito adverso para o guaru. Os sinais de intoxicação do guaru podem ser um indicativo de contaminação ambiental por essas moléculas.
\end{abstract}

Palavras-chave: ecotoxicologia, peixe, qualidade de água, sinais de intoxicação.

Ecotoxicity of agricultural surfactants for guaru Phalloceros caudimaculatus

\begin{abstract}
The surfactants are amphipathic molecules that promote the reduction of surface tension of water. The objectives were to estimate the LC(I)50;96h; to determine the assessment risk;to assess the water quality and the intoxication signs during

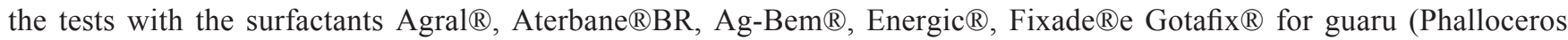
caudimaculatus). The animals were exposed to the surfactants and a control treatment in completely randomized design with three replicates. To characterize the assessment risk were used the data of the estimated environmental concentration and of the LC(I)50;96 hours of each surfactant, classifying them according to a coefficient of risk. A LC(I)50;96h of surfactant Agral ${ }^{\circledR}$ was $4.33 \mathrm{mgL}-1$; Aterbane ${ }^{\circledR B R} 5.81 \mathrm{mgL}-1$; Energic ${ }^{\circledR} 3.93 \mathrm{mgL}-1$; GOTAFIX ${ }^{\circledR} 3.40 \mathrm{mgL}-1$; Fixade ${ }^{\circledR} 4.06 \mathrm{mgL}-1$ and Ag-Bem ${ }^{\circledR 2} 2.19 \mathrm{mgL}-1$. The water quality was not changed by the presence of surfactants. The intoxication signs showed were: increase in opercullar beat, loss of ability to pitching, erratic swimming and spasms. Ag-Bem ${ }^{\circledR}$ can be considered as slightly toxic and anothers moderately toxic. Aterbane ${ }^{\circledR} B R$, Agral ${ }^{\circledR}, A g-B e m \AA$ and Gotafix ${ }^{\circledR}$ are considered at risk with possibility adverse effect and Energic ${ }^{\circledR}$ and Fixade ${ }^{\circledR}$ risk likely adverse effect to the guaru. The intoxication signs of guaru may be indicative of environmental contamination by these molecules.
\end{abstract}

Keywords: ecotoxicology, fish, water quality, intoxication signs.

*Corresponding author: Silvia Patrícia Carraschi, e-mail: pacarraschi@yahoo.com.br 


\section{INTRODUÇÃO}

Os surfactantes fazem parte dos componentes inertes das formulações dos herbicidas. Estas substâncias são moléculas anfipáticas que melhoram a aderência dos herbicidas e facilitam a penetração do ingrediente ativo nas plantas (Wagner et al., 2003). Porém esses compostos podem ser mais tóxicos que o próprio ingrediente ativo, para organismos nãoalvos (Oakes \& Pollak, 2000). Estima-se que cerca de 100 mil desses compostos são produzidos, equivalente a 200 milhões de toneladas por ano, dos quais 20 a $30 \%$ podem chegar aos ambientes aquáticos (Colla \& Costa, 2003).

Os surfactantes causam danos nos componentes lipídicos das membranas celulares e a diminuição da tensão superficial da água, reduz a hibrofobicidade dos lipídeos da célula, causando uma superidratação. Em altas concentrações pode ocorrer supressão dos processos metabólitos evoluindo a necrose celular (FAO, 1993).

Devido à utilização em diversos setores agroindustrial e doméstico, vários trabalhos foram realizados para avaliar a toxicidade dos surfactantes para os peixes: Lepomis macrochirus (Macek \& Krzeminski, 1975); Brachidanio rerio e Oncorhynchus mykiss (Fogels \& Sprague, 1977); L. macrochirus (Singer et al., 1994 e 1995); Macquaria novemaculeata (Gullec \& Holdway, 2000); L. macrochirus (Buhl \& Hamilton, 2000); Centropomus parallelus (Rocha et al., 2007) e Pimephales promelas (Teneyke \& Markee, 2007).

No Brasil, para o desenvolvimento de novos produtos (herbicidas, para a agricultura ou antibióticos e parasiticidas para aquicultura) é necessário o estudo de avaliação do risco e da periculosidade que o produto pode oferecer para o ambiente. Dessa avaliação resulta uma classificação que indica o perfil ecotoxicológico do produto e fornece instruções para o uso correto (IBAMA, 1987).

Os testes de toxicidade são etapas iniciais de avaliação de risco de xenobióticos, proporcionando a caracterização da resposta ecológica a um elemento químico (Cairns et al., 1998).

Para esses testes são utilizados diversas espécies representantes da cadeia trófica. Nesse trabalho foi utilizado o guaru (Phalloceros caudimaculatus), uma espécie ovovivípara, com dimorfismo sexual pertencente à ordem Ciprinodontiforme que habita riachos e lagoas (Casatti et al., 2001), e possui as características de um bom organismoteste, tais como: representatividade de um grupo ecológico; fácil disponibilidade para obtenção; ciclo reprodutivo curto, vivíparo, cosmopolita e é uma espécie nativa do Brasil (Wolff et al., 2007).

Assim, os objetivos deste trabalho foram: estimar a $\mathrm{CL}(\mathrm{I})_{50} ; 96 \mathrm{~h}$ do surfactante Aterbane ${ }^{\circledR}$ BR (mistura de condensado de alcoolfenóis com óxido eteno e sulfonados orgânicos), Agral $^{\circledR}$, Energic ${ }^{\circledR}$ (nonil fenoxipoli (etilenoxi) etanol), Ag-Bem ${ }^{\circledR}$ (agente tenso-ativo aniônico), Fixade ${ }^{\circledR}$ (nonil fenol etoxilato) e de Gotafix ${ }^{\circledR}$ (nonil fenol polietileno glicol éter) para o peixe guaru. Avaliar as variáveis de qualidade de água; os sinais de intoxicação e o risco ambiental desses compostos para essa espécie.

\section{MATERIAL E MÉTODOS}

\section{Condições ambientais e experimentais dos testes de toxicidade aguda}

Os experimentos foram realizados no Laboratório de Impacto Ambiental do Núcleo de Estudos e Pesquisas Ambientais em Matologia (NEPEAM), da Faculdade de Ciências Agrárias e Veterinárias, UNESP, Campus de Jaboticabal.

Os peixes, machos e fêmeas, foram coletados de viveiros de criação de camarão (Macrobrachium amazonicum) do Centro de Aquicultura da Unesp de Jaboticabal. Os organismos ficaram por cerca de 40 dias em tanques de $400 \mathrm{~L}$, em aclimatação no setor de cultivo do laboratório, recebendo ração comercial a vontade uma vez ao dia. Não foi observado mortalidade desse lote de organismo teste.

Os organismos teste com peso entre 0,3 a 0,6 gramas foram transferidos para tanques de $250 \mathrm{~L}$ em sala climatizada, com temperatura média de $27 \pm 2{ }^{\circ} \mathrm{C}$ e fotoperíodo diário de $12 \mathrm{~h}$ de luz em que foram aclimatados por dez dias. A aclimatação ocorreu em sistema de aeração constante e os animais foram alimentados à vontade com ração comercial (contendo 32\% $\mathrm{PB}$, uma ração recomendada para peixes jovens), uma vez ao dia.

Primeiramente, para avaliar a sanidade dos peixes, foram realizados testes de sensibilidade usando como substânciareferência o cloreto de potássio (KCl) (ABNT, 2004), sendo a $\mathrm{CL}(\mathrm{I})_{50} ; 96 \mathrm{~h}$ média estimada em $0,55 \pm 0,04 \mathrm{~g} \mathrm{~L}^{-1}$.

Inicialmente, para a realização dos testes definitivos, foram realizados testes preliminares de toxicidade aguda determinando o intervalo de concentração que causa zero e $100 \%$ de mortalidade.

Os testes foram conduzidos em sistema estático, em delineamento inteiramente casualizado (DIC) nas seguintes condições laboratoriais: $\mathrm{pH}$ entre 7,0 e 8,0; oxigênio dissolvido entre 5,0 e 6,0 $\mathrm{mgL}^{-1}$; condutividade elétrica da água entre 170,0 e $180,0 \mu \mathrm{Scm}^{-1}$; temperatura da sala entre 27 e $29^{\circ} \mathrm{C}$; e temperatura da unidade experimental entre 25 e $26^{\circ} \mathrm{C}$ (ABNT, 2004).

A avaliação da mortalidade foi diária com a retirada dos peixes mortos dos aquários. Os aquários em que os testes foram realizados eram de vidro com capacidade para $5 \mathrm{~L}$.

\section{Testes definitivos e avaliação do comportamento dos peixes}

A partir dos dados obtidos nos testes preliminares, foram realizados os testes definitivos, utilizando as seguintes concentrações dos surfactantes: Ag-Bem ${ }^{\circledR}: 15,0 ; 18,0 ; 21,0$; 24,$0 ; 27,0 ; 30,0 \mathrm{mgL}^{-1} ; \mathrm{Agral}^{\circledR}: 2,0 ; 3,0 ; 4,0 ; 5,0 ; 6,0 ; 7,0 \mathrm{mgL}^{-}$ 
1; Aterbane ${ }^{\circledR}$ BR: 3,0; 4,0; 5,0; 6,0; 7,0;8,0 $\mathrm{mgL}^{-1}$; Energic ${ }^{\circledR}$ : 3,$0 ; 3,5 ; 4,0 ; 4,5 ; 5,0 \mathrm{mgL}^{-1}$; Fixade ${ }^{\circledR}: 2,0 ; 4,0 ; 6,0 ; 8,0 \mathrm{mgL}^{-1}$ e Gotafix ${ }^{\circledR}: 1,0 ; 3,0 ; 5,0 ; 7,0 \mathrm{mgL}^{-1}$, mais um controle, sem adição de surfactante, com três réplicas por tratamento.

Durante a realização dos testes foram avaliadas as variáveis de qualidade da água: $\mathrm{pH}$, temperatura, oxigênio dissolvido $\left(\mathrm{mgL}^{-1}\right)$ e condutividade elétrica $\left(\mu \mathrm{Scm}^{-1}\right)$ em 0,24 e 48 horas após a exposição aos surfactantes.

Os sinais de intoxicação também foram avaliados, sendo observados: batimento opercular, busca por oxigênio, capacidade de arfagem, natação errática e posição na coluna de água (Murty, 1988), em 0, 4, 24, 48, 72 e 96 horas após a exposição aos surfactantes.

Em cada réplica de cada tratamento foram utilizados cinco peixes e a avaliação da mortalidade foi diária com a retirada dos peixes mortos.

Ao final de 96 horas, com os dados de mortalidade, foram calculados os valores da CL(I $)_{50} ; 96 \mathrm{~h}$ pelo software Trimmed Sperman Karber (Hamilton et al., 1977).

\section{Avaliação do Risco Ambiental dos surfactantes}

A classificação do risco ambiental dos surfactantes foi realizada por meio do método do quociente $(\mathrm{Q})$ citada por Urban \& Cook (1986). O valor do Q foi calculado pela divisão dos valores da concentração ambiental estimada (CAE) pela $\mathrm{CL}(\mathrm{I})_{50} ; 96 \mathrm{~h}$ de cada surfactante.

A concentração ambiental estimada de cada surfactante foi baseada nas recomendações agronômicas (dosagem de campo) do Ministério da Agricultura Pecuária e Abastecimento (MAPA) e descrita na ficha técnica (FISPQ) de cada produto. A partir desta dosagem obteve-se a concentração de ingrediente ativo em cada aplicação do produto, sendo esta considerada a CAE.

As classes de risco ambiental utilizadas foram: risco com nenhum efeito adverso se $\mathrm{Q} \leq 0,1$; Risco com possibilidade de efeito adverso se $0,1 \leq \mathrm{Q} \leq 10$; e Risco com probabilidade de efeito adverso se $\mathrm{Q}>10$.

\section{RESULTADOS E DISCUSSÃO}

Os surfactantes não alteraram as variáveis de qualidade de água, permanecendo dentro dos parâmetros iniciais recomendados pela ABNT (2004) para testes de toxicidade, ou seja, $\mathrm{pH}$ entre 7,0 e 8,0; oxigênio acima de $4,0 \mathrm{mgL}^{-1} \mathrm{e}$ condutividade entre 130 e $140 \mu \mathrm{Scm}^{-1}$.
A CL(I) $)_{5} ; 96 \mathrm{~h}$ dos surfactantes variou de $3,40 \mathrm{mgL}^{-1}$ $\left(\right.$ Gotafix $\left.^{\circledR}\right)$ à 22,19 $\mathrm{mgL}^{-1}\left(\mathrm{Ag}^{-B e m}{ }^{\circledR}\right)$ (Tabela 1$)$.

Os surfactantes Energic $^{\circledR}$, Agral ${ }^{\circledR}$, Gotafix ${ }^{\circledR}$, Aterbane ${ }^{\circledR}$ BR e Fixade ${ }^{\circledR}$ podem ser classificados como moderadamente tóxicos $\left(\mathrm{CL}_{50}\right.$ entre 1 e $\left.10 \mathrm{mgL}^{-1}\right)$ e o $\mathrm{Ag}-\mathrm{Bem}^{\circledR}$ como pouco tóxico $\left(\mathrm{CL}_{50}\right.$ entre 10 e $\left.100 \mathrm{mgL}^{-1}\right)$ (Zucker, 1985).

Para o $\mathrm{Ag}-\mathrm{Bem}^{\circledR}$ nas concentrações 0,0 e $15,0 \mathrm{mgL}^{-1}$ não ocorreram mortalidades; em $18,0 \mathrm{mgL}^{-1}$ a mortalidade foi de $33,33 \%$; em 21,0 e $24,0 \mathrm{mgL}^{-1}, 46,67 \%$; em $27,0 \mathrm{mgL}^{-1}$, $60,0 \%$; e em $30,0 \mathrm{mgL}^{-1}, 100,0 \%$. Para os demais surfactantes a relação concentração-mortalidade está apresentada na figura 1. Nos controles de todos os testes de toxicidade de todos os surfactantes não foi observado mortalidade dos organismos teste.

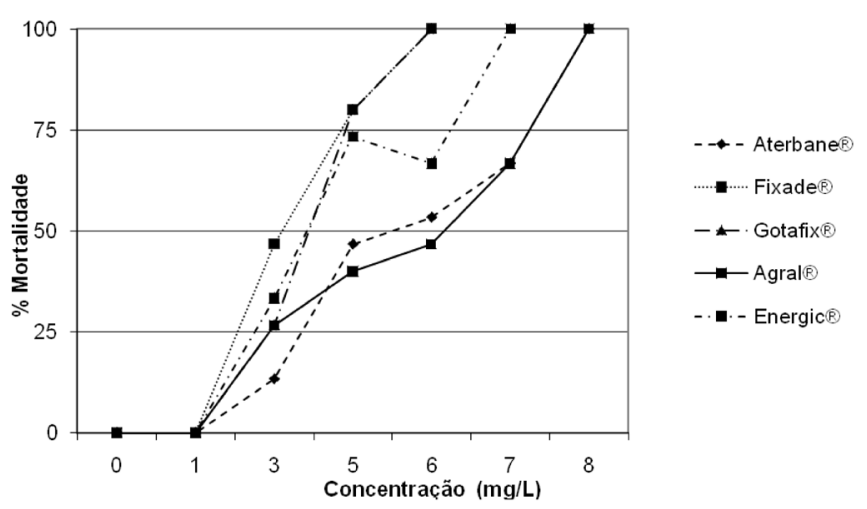

Figura 1 - Relação concentração-mortalidade durante os testes de toxicidade aguda dos cinco surfactantes.

As toxicidades dos surfactantes testados e de outros surfactantes encontrados na literatura estão apresentadas na tabela 2 .

A toxicidade de surfactantes não iônicos pode estar relacionada com o comprimento da cadeia carbônica, sendo que estruturas moleculares com mais de 12 carbonos afetam com maior intensidade a permeabilidade da membrana plasmática (Talmage, 1994), ocorrendo assim, uma maior absorção do xenobiótico. Os surfactantes Energic ${ }^{\circledR}$ e o Agral $^{\mathbb{R}}$ possuem 35 carbonos na sua estrutura molecular, podendo ser esse um dos motivos para explicar sua toxicidade.

A toxicidade dos surfactantes não-iônicos também poderia ser explicada por impedir a respiração celular, pois causam disfunção endócrina e lise celular das membranas epiteliais branquiais (McWillians \& Payne, 2001).

Tabela 1 - Toxicidade aguda dos surfactantes para o guaru.

\begin{tabular}{|c|c|c|c|c|c|c|}
\hline & \multicolumn{6}{|c|}{ Surfactantes $\left(\mathrm{mgL}^{-1}\right)$} \\
\hline & Agral $^{\circledR}$ & Aterbane $^{\circledR}$ BR & Gotafix $^{\circledR}$ & Energic $^{\circledR}$ & Fixade $^{\circledR}$ & Ag-Bem ${ }^{\circledR}$ \\
\hline LI & 3,84 & 5,33 & 2,77 & 3,72 & 3,47 & 20,69 \\
\hline $\mathrm{CL}(\mathrm{I})_{50} ; 96 \mathrm{~h}$ & 4,33 & 5,81 & 3,40 & 3,93 & 4,06 & 22,19 \\
\hline LS & 4,90 & 6,36 & 4,17 & 4,14 & 4,76 & 23,80 \\
\hline
\end{tabular}

$\mathrm{LS}=$ limite de superior; $\mathrm{CL}(\mathrm{I})_{50} ; 96 \mathrm{~h}=$ concentração letal $50 \% ; \mathrm{LI}=$ limite inferior 
Para os surfactantes iônicos, como o Aterbane ${ }^{\circledR}$ BR, a toxicidade pode ser atribuída a capacidade desses compostos de aumentar a permeabilidade de membrana, alterando as propriedades físicas da bicamada lipídica, permitindo a entrada de uma maior quantidade de íons (Pretti et al., 2006). Além disso, segundo os mesmos autores, os surfactantes iônicos Ammoeng $100^{\circledR}$ e Ammoeng130 ${ }^{\circledR}$ causaram descamação epitelial e hipertrofia, hiperplasia; desorganização da lamela secundária e vacuolização citoplasmática, nas brânquias, levando o animal a morte.

Segundo Hofer et al. (1995) os surfactantes aniônicos causam hiperplasia epitelial, aumentando a distância interlamelar e dificultando a difusão do oxigênio, o que resulta em estresse respiratório e falha osmoregulatória. Assim, essa alteração pode ser um dos motivos para explicar a toxicidade do Ag-Bem ${ }^{\circledR}$, Gotafix ${ }^{\circledR}$ e Fixade ${ }^{\circledR}$.

Durante as avaliações em 0, 4, 24, 48, 72 e 96 horas após o início da exposição, os animais apresentaram agitação, perda da capacidade de arfagem, aumento do batimento opercular, natação errática e comportamento letárgico. Observou-se mortalidade durante todo o período experimental.

Segundo a FAO (1993) os sinais clínicos de intoxicação por surfactantes incluem: desordens respiratórias (aumento da taxa respiratória e busca por oxigênio na superfície) e posteriormente inatividade.

De acordo com Lewis (1991) a alteração da freqüência opercular é um dos efeitos de surfactantes aniônicos, pois esses compostos provocam acúmulo de muco na superfície do epitélio branquial, prejudicando a difusão dos gases.

O aumento do batimento opercular observado no guaru foi semelhante ao comportamento apresentado pela tainha (Mugil platanus) exposta à sulfobato alquilbezeno linear (LAS) (Barbieri, 2005).

A natação errática, a perda do equilíbrio e a diminuição de atividade também foi verificada em concentrações de $10 \mathrm{mgL}^{-1} \mathrm{de}$ surfactantes iônicos (Pretti et al., 2006) e o aumento da concentração do LAS-C12 também causou diminuição da capacidade de natação de Cyprinus carpio (Barbieri et al., 2005).

Quanto à classificação do risco ambiental dos surfactantes, o Aterbane ${ }^{\circledR} \mathrm{BR}$, o $\mathrm{Agral}^{\circledR}$, o Ag-Bem ${ }^{\circledR}$ e o Gotafix ${ }^{\circledR}$ podem ser considerados de risco com possibilidade efeito adverso $(0,01 \leq \mathrm{Q} \leq 10)$ e o Energic $^{\circledR}$ e o Fixade $^{\circledR}$ de risco com probabilidade efeito adverso $(\mathrm{Q} \leq 10)$ para o guaru (Tabela 2).

O Agral ${ }^{\circledR}$; o Aterbane ${ }^{\circledR}$ BR; o Ag-Bem ${ }^{\circledR}$ e o Gotafix ${ }^{\circledR}$ apresentaram o mesmo risco ambiental $(0,1 \leq \mathrm{Q} \leq 10)$ que

Tabela 2 - Toxicidade de surfactantes para várias espécies de peixes.

\begin{tabular}{|c|c|c|c|}
\hline Surfactantes & $\mathrm{CL}_{50} ; 96 \mathrm{~h}\left(\mathrm{mgL}^{-1}\right)$ & Espécie & Referência \\
\hline Agral $^{\circledR}$ & 4,33 & \multirow{6}{*}{ P. caudimaculatus } & \multirow{6}{*}{ Presente trabalho } \\
\hline Aterbane ${ }^{\mathbb{R}} \mathrm{BR}$ & 5,81 & & \\
\hline Gotafix $^{\circledR}$ & 3,40 & & \\
\hline Energic $^{\circledR}$ & 3,93 & & \\
\hline Fixade $^{\circledR}$ & 4,06 & & \\
\hline Ag-Bem ${ }^{\circledR}$ & 22,19 & & \\
\hline Corexit 9527 & 0,08 & Lepomis macrochirus & Singer et al., 1994 \\
\hline LI700 & 210,0 & L. macrochirus & Rubin, 1997 \\
\hline \multirow[t]{2}{*}{ LI700 } & 130,0 & Oncorhynchus mykiss & Rubin, 1997 \\
\hline & & O. mykiss; & \\
\hline \multirow[t]{2}{*}{ Agri-Dex ${ }^{\circledR}$} & $>1000$ & & Rubin, 1997 \\
\hline & & L. macrochirus & \\
\hline Corexit 9527 & 14,30 & Macquaria novemaculeata & Gulec \& Holdway, 2000 \\
\hline Dodecil sulfato de sódio & 24,90 & O. mykiss & Buhl \& Hamilton, 2000 \\
\hline $\begin{array}{l}\text { Dodecil sulfato benzeno } \\
\text { de sódio (SDBS) }\end{array}$ & 33,61 & Salmo gairdneri & Singh et al., 2002 \\
\hline SDBS & 40,15 & Gammbusia affinis & Singh et al., 2002 \\
\hline SDBS & 38,04 & Carassius auratus & Singh et al., 2002 \\
\hline Aterbane ${ }^{\circledR} \mathrm{BR}$ & 10,80 & Brachydanio rerio & Dow Agroscience, 2006 \\
\hline Gotafix $^{\circledR}$ & 0,190 & B. rerio & Dow Agroscience, 2006 \\
\hline Nonilfenol (NP) & 0,14 & Pimephales promelas & Teneyke \& Markee, 2007 \\
\hline
\end{tabular}

Tabela 3 - Avaliação de risco ambiental dos surfactantes baseada na concentração ambiental estimada (CAE).

\begin{tabular}{|c|c|c|c|c|}
\hline Surfactantes & $\begin{array}{c}\text { Dosagens utilizadas em } \\
\text { campo }\end{array}$ & $\mathrm{CAE}(\mathrm{g} / \mathrm{mL})$ & $\mathrm{Q}=\mathrm{CAE} / \mathrm{CL}_{50}$ & Risco Ambiental \\
\hline Agral $^{\circledR}$ & 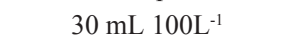 & 6,0 & 1,34 & Possibilidade de efeito adverso \\
\hline Aterbane ${ }^{\circledR} \mathrm{BR}$ & 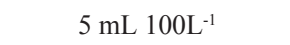 & 2,3 & 0,40 & Possibilidade de efeito adverso \\
\hline Ag-Bem ${ }^{\circledR}$ & 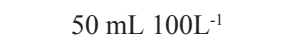 & 6,5 & 0,30 & Possibilidade de efeito adverso \\
\hline Energic $^{\circledR}$ & 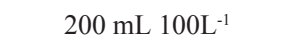 & 45,2 & 11,50 & Probabilidade efeito adverso \\
\hline Fixade $^{\circledR}$ & $200 \mathrm{~mL} \mathrm{100 \textrm {L } ^ { - 1 }}$ & 180,0 & 44,33 & Probabilidade efeito adverso \\
\hline Gotafix $^{\circledR}$ & $60 \mathrm{~mL} \mathrm{100 \textrm {L } ^ { - 1 }}$ & 7,5 & 2,21 & Possibilidade de efeito adverso \\
\hline
\end{tabular}


o sabão e o LAS para vários organismos aquáticos (bactéria, crustáceo, inseto, peixe e anfíbios) (Van de Plassche et al., 1997; Mungray \& Kumar, 2008). Porém apresentaram menor risco ambiental que o álcool etoxilato (AE) e o álcool etoxilato sulfatado (AES) $(\mathrm{Q} \leq 0,1)$ para organismos aquáticos (Van de Plassche et al., 1997).

No entanto, o Energic ${ }^{\circledR}$ e o Fixade $^{\circledR}$ apresentaram maior risco ambiental (Q > 10) que o sabão, o LAS, o AE e o AES (Van de Plassche et al., 1997; Mungray \& Kumar, 2008).

Os surfactantes não causam alteração nas variáveis de qualidade de água. O Energic ${ }^{\circledR}$ e o Fixade $^{\circledR}$ apresentam um maior risco ambiental que os demais surfactantes e o Ag-Bem foi o menos tóxico para $P$. caudimaculatus.

Agradecimentos: À Fundação de Amparo à Pesquisa do Estado de São Paulo (FAPESP), Bolsa de Iniciação Científica do primeiro autor, proc $\mathrm{n}^{\circ}: 2006 / 58459-5$.

\section{REFERÊNCIAS}

ABNT. Associação Brasileira de Normas Técnicas, 2004, NBR 15088: Ecotoxicologia aquática - Toxicidade aguda - Método de ensaio com peixes. São Paulo, 19p.

BARBIERI, E, 2005, Efeito do LAS-C12 (dodecil benzeno sulfonato de sódio) sobre alguns parâmetros do comportamento da tainha (Mugil platanus), Atlânt., Rio Grande, 27(1): 49-57.

BUHL, K., HAMILTON, S., 2000, Acute toxicity to fire-control chemicals, nitrogeneous chemicals, and surfactants to rainbow trout. Trans. Amer. Fish. Soci, 129: 408-418. doi: 10.1577/15488659(2000)129<0408:ATOFCC $>2.0 . C O ; 2$.

CAIRNS, J. J., NIEDERLEHNER, B. R., BIDWELL, J. R., 1998, Ecological toxicity testing. IN: MEYERS, R. A. Encyclopedia of Environmental Analysis and Remediation, John Wiley e Sons, Inc., New York, 1560p.

CASATTI, L., LANGEANI, F., CASTRO, R. M. C., 2001, Peixes de riacho do parque estadual Morro do Diabo, Bacia do Alto Rio Paraná, SP., Biot. Neot., 1: 1-15.

COLLA, L. M., COSTA, J. A. V., 2003, Obtenção e aplicação de biossurfactantes. Vetor, 13: 85-103.

DOW AGROSCIENCE, 2006, Ficha de Informação de Segurança de Produto Químico

- FISPQ - ATERBANE ${ }^{\circledR} B R$, São Paulo, 1-8p.

FAO, Food and Agriculture Organization of the United Nations, 1993. Svobodová, Z.; Lloyd, R.; Máchová, J.; Vykusová, B. Water quality and fish health. EIFAC Technical Paper, $\mathrm{n}^{\circ}$ 54, Rome, $\mathrm{p} 59$.

FOGELS, A. I., SPRAGUE, J. B., 1977, Comparative Short-Term Tolerance of Zebrafish, Flagfish, and Rainbow Trout to Five Poisons Including Potential Reference Toxicants. Wat. Res., 11: 811-817. doi:10.1016/0043-1354(77)90052-5.

GULLEC, I., HOLDWAY, D. A., 2000, Toxicity of crued oil and dispersed crude oil to ghost shrimp Palaemon serenus and larvae of Australian bass Macquaria novemaculeata., Environ. Toxic., 15(2): 91-98.

HAMILTON, M. A., RUSSO, R. C., THURSTON, V., 1997, Trimed Sperman-Karber method for estimating medial lethal concentrations in toxicology bioassays. Environ. Scien. Technol., Iowa, 7: 714-719.

HOFER, R., JENEY, Z., BUCHER, F., 1995, Chronic effects of linear alkylbenzene sulfonate (LAS) and ammonia on rainbow trout (Oncorhynchus mykiss) fry at water criteria limits. Water Res., New York, 29: 2725-9. doi: 10.1016/00431354(95)00143-9.

IBAMA, 1987, Avaliação da toxicidade aguda para peixes. Parte D. 3. Manual de testes para avaliação de ecotoxicidade de agentes químicos, p. D.3.2., 01/09.

LEWIS, M. A, 1991, Chronic and sublethal toxicities of surfactants to aquatic animals: a review and risk assessment. Wat. Res., 25: 101-113. doi:10.1016/0043-1354(91)90105-Y.

MACEK, J. K., KRZEMINSKI, S. F., 1975, Susceptibility of bluegill sunfish (Lepomis macrochirus) to nonionic surfactants. Bullet. Environ. Contamin. \& Toxic., 13(3): 377-384. doi: 10.1007/ BF01685354.

McWILLIANS, P., PAYNE, G., 2001, Bioaccumulation potential of surfactants: a review. Roy. Soci. Chem. \& EOSCA, Manchester, 2: 13 .

MUNGRAY, A. K., KUMAR, P., 2008, Occurrence of anionic surfactants in treated sewage: Risk assessment to aquatic environment. J. Hazar. Mater., 160. doi:10.1016/j. jhazmat.2008.03.025.

MURTY, A. S., 1988, Toxicology of pesticide to fish. Boca Raton, Editora: CRC Press., v.1, 129p.

OAKES, D. J., POLLAK, J. K., 2000, The in vitro evaluation of toxicities of there realated herbicide formulation containing ester derivatives 2(4,5): 2,4 using sub mitochondrial particles. Toxicol., Limerick, 151: 1-9. doi:10.1016/S0300-483X(00)00244-4.

PRETTI, C., CHIAPPE, C., PIERACCINI, D., GREGORI, M., ABRAMO, F., MONNI, G., INTORRE, L., 2006, Acute toxicity of ionic liquids to the zebrafish (Danio rerio). Green Chem., 8: 238-240. doi: 10.1039/B511554J.

ROBERTS, D. W., MARSHALL, S. J., 1995, Application of hydrophobicity parameters to prediction of the acute aquatic toxicity of commercial surfactant mixtures. SAR QSAR Environ. Res., 4: 167-176. doi: 10.1080/10629369508029914.

ROCHA, A. J. S., GOMES, V., NGAN, P. V., PASSOS, M. J. A. C. R., FURIA, R. R., 2007, Effects of anionic surfactant and salinity on the bioenergetics of juveniles of Centropomus parallelus (Poey). Ecotox. Envir. Saf., New York, 68: 397-404. doi:10.1016/j.ecoenv.2006.10.007.

RUBIN, L., 1997, Effects of surfactants on the toxicity of glyphosate, wit. spec. Refer. Rod., $28 \mathrm{p}$.

SINGER, M. M., GEORGE, S., JACOBSON, S., LEE, I., TJEERDEMA, R. S., SOWBY, M. L., 1994, Comparative Toxicity of Corexit ${ }^{\circledR} 7664$ to the early life stages of four marine species. Arch. Envir. Contam. Toxic., New York, 27: 130-136. doi: 10.1007/BF00203899.

SINGER, M. M., GEORGE, S., JACOBSON, S., LEE, I., WEETMAN, L. L., TJEER DEMA, R. S.; SOWBY, M. L., 1995, Acute toxicity of the oil dispersant Corexit 9554 to marine organisms., Ecotox. Envir. Saf., New York, 32: 81-86. doi:01476513/95.

SINGH, R. P., GUPTA, N., SINGH, S., SINGH, A., SUMAN, R., ANNIE, K., 2002, Toxicity of ionic and non-ionic surfactants to six microbes found in Agra, India. Bull. Envir. Contam. Toxicol., 69: 265-270. doi: 10.1007/s00128-002-0056-z.

TALMAGE, S. S., 1994, Environmental and human safety of major surfactants: alcohol ethoxylates and alkylphenol ethoxylates. Lewis publishers, Boca Raton, FL, USA, 374p.

TENEYKE, M. C., MARKEE, T. P., 2007, Toxicity of Nonylphenol, Nonylphenol Monoethoxylate, and Nonylphenol Diethoxylate and Mixtures of these Compounds to Pimephales promelas (Fathead Minnow) and Ceriodaphnia dubia. Arch. Environ. Contam. Toxic., New York, 53: 599-606. doi: 10.1007/s00244- 
006-0249-2

URBAN, D. J., COOK, N. J. 1986, Hazard evaluation division Standard evaluation procedure ecological risk assessment, Washington, USEPA Public. 540/9: 86-001.

VAN de PLASSCHE, E., BRUIJIN, J., BILTHOVEN, FEIJTEL, T., BEVER, S,1997, Risk assessment of four major surfactants groups in the Netherlands. Tenside Surfac. Deterg., 34: 40. doi: 35400006892955.0050 .

WAGNER E. D., MARENGO, M. S., PLEWA, M. J, 2003, Modulation of the mutagenicity of heterocyclic amines by organophosphate insecticides and their metabolites. Mutation Res., 536: 103-115. doi:10.1016/S1383-5718(03)00037-8.

WOLFF, L. L.; HRECIUK, E. R.; VIANA, D.; ZALESKI, T.; DONATTI, L. 2007, Population structure of Phalloceros caudimaculatus(Hensel, 1868)(Cyprinodontiformes,Poeciliidae) collected in a brook in Guarapuava, PR. Braz. Arch. Biol. Tech., 50 (3): 417-423 doi: 10.1590/S1516-89132007000300008.

ZUCKER, E, 1985, Hazard Evaluation Division - Standard Evaluation Procedure - Acute Toxicity Test for Freshwater Fish. USEPA Publication, 540/9- 85-006, Washington, 17p. 